\title{
Análise do avanço das equipes de saúde bucal inseridas na Estratégia Saúde da Família em Pernambuco, região Nordeste, Brasil, 2002 a 2005
}

\author{
Analysis of the progress of the oral health teams \\ inserted in the Family Health Strategy in Pernambuco, \\ Northeast Region, Brazil, 2002 until 2005
}

\author{
Shirley Florêncio da Silva ${ }^{1}$ \\ Petrônio J osé de Lima M artelli ${ }^{1}$ \\ Domício Aurélio de Sá ${ }^{1}$ \\ Amanda Priscila de Cabral ${ }^{1}$ \\ Fernando Castim Pimentel ${ }^{1}$ \\ Ive da Silva M onteiro ${ }^{1}$ \\ Cícera Lissandra Sá V ieira M acedo ${ }^{1}$
}

\footnotetext{
${ }^{1}$ Departamento deSaúde

Coletiva, Centro de

Pesquisas Aggeu

M agalhães. Rua AndréGide 53, Bairro Encruzilhada. 52041-521 Recife PE.

Abstract The aim of this study was to analyze the implementation of oral health care in the Family Health Strategy in the State of Pernambuco, Brazil, 2002 until 2005. It was observed aspects regarding the expansion of the teams, the percentage of population covered and the rise in the quantitative of oral primary procedures. It was a descriptive and quantitative study, focusing on the 185 cities included in the Family $\mathrm{H}$ ealth Strategy and all cities that included an oral health care in the program. The results showed that the evaluation of the oral health teams was $204.22 \%$, whilethe percentage of theevolution of oral health care procedures $19.19 \%$. It was observed that the expansion of thetypel $(327,14 \%)$ washigher than type II (125,92\%). The estimation of the population covered by oral health team was about $29.56 \%$ to 3.450 inhabitants (D ecreen. 673/03). The financing of oral health care primary had an increase in the same period of $389.50 \%$. At last, it was verified, in practice, the oral care existent service to oral primary care procedures were changed in oral health teams.

Key words Primary health care, Family H ealth Strategy, Oral health
Resumo Aborda-se neste estudo a evolução da saúde bucal, de dezembro de 2002 a dezembro de 2005, na Estratégia Saúde da Família em Pernambuco, Brasil. O bservaram-se os aspectosquanto à expansão das equipes, ao percentual de cobertura e à elevação no quantitativo dos procedimentos odontológicos. Foi um estudo descritivo, exploratório e deabordagem quantitativa. 0 universo se constituiu dos 185 municípios do estado e a amostra comportou todos os municípios inseridos na Saúdeda Família contemplados com saúde bucal. Os resultados mostraram que a evolução das equipes de saúde bucal foi de $204,22 \%$, enquanto o percentual de evolução de procedimentos odontológicos foi de 19,19\%. O bservou-se que a expansão da modalidade tipo I $(327,14 \%)$ se deu mais intensamente que a tipo II (125,92\%). A estimativa da cobertura ficou em $29,56 \%$ para 3.450 habitantes (Portaria $\mathrm{n}$ - 673/03). Q uanto ao financiamento das equipes, a evolução do incentivo repassado à saúde bucal foi de $389,50 \%$. Por fim, verificou-se que, na prática, os serviços existentes de assistência odontológica para atenção básica foram transformados em equipes de saúde bucal.

Pal avras-chave Atenção básica, Estratégia Saúde da Família, Saúde bucal 
Introdução

A saúde, no país, adquiriu visibilidade com a Constituição Federal de 1988, a qual instituiu a saúde como um direito de todos e um dever do Estado, operada por meio do Sistema Ú nico de Saúde (SUS). N os níveis federal, estadual e municipal, os gestores dessesistema vêm atribuindo ênfase à mudança do modelo de atenção à saúde, priorizando a Atenção Básica.

N essa perspectiva de construção de políticas públicas de saúde que possam garantir a integralidade do atendimento, sem o qual uma política de saúdenão podeser pensada, todas as ações relativas à atenção básica confluem para a processo de expansão do Programa Saúde da Família (PSF), incentivada pelo M inistério da Saúde $(\mathrm{MS})^{1}$

Estudar o percurso realizado pela saúde bucal na atenção primária é importante no sentido de entender a forma pela qual a odontologia se inseriu estruturalmente no processo de produção do modelo de atenção à saúde atual. Essa organização e a definição da prática da saúde bucal necessitam de uma reflexão teórica de sua organização.

Pode-se afirmar que a saúde bucal no Brasil acumulou saberes e práticas que vão desde sua inserção nas Caixas de Aposentadoria, passando pelo Sistema Unificado e Descentralizado de Saúde (Suds) e, finalmente, chegando ao SUS².

O PSF, hoje melhor denominado de Estraté gia Saúde da Família, criado pelo MS em 1994, vem mostrando sua efetividade como estratégia de reorganização do modelo de atenção à saúde, procurando atuar de forma ampla e integralizada. A Lei n 8.080 , de $1990^{3}$, regulou as ações e os serviços desaúde, estabeleceu os princípios ediretrizes do SUS que norteiam e sustentam o PSF. N esse aspecto, o indivíduo e a família são identificados não apenas como usuários do serviço de saúde, mas como sujeitos participativos para a consolidação do conceito de saúde como caráter coletivo e de qualidade de vida.

O PSF apresentou-se como uma possibilidade de reestruturação da atenção básica, a partir de um conjunto de ações conjugadas em sintonia com os princípios de territorialização, intersetorialidade, descentralização, corresponsabilização e priorização de grupos populacionais com maior risco de adoecer ou morrer. As bases dessa estratégia destacam que, ao contrário do modelo tradicional, centrado na doença e no hospital, o PSF prioriza as ações de proteção e promoção à saúde dos indivíduos e da família, tanto adultos quanto crianças, sadios ou doentes, de forma integral e contínua 4 .

O financiamento da saúde bucal no SUS em nível federal -, com o repasse de recursos aos estados e municípios para financiar as ações de saúde bucal, está inserido no Piso da Atenção Básica (PAB), definido pela N orma O peracional Básica (NOB) 96, o que representou o principal avanço no processo de descentralização do SUS ocorrido nos últimos anos 5 .

A proposta de ampliação da atenção básica trazida pela N orma Organizacional de Assistência à Saúde (Noas/01) buscou definir inequivocamente as responsabilidades e ações estratégicas mínimas que todos os municípios brasileiros deveriam desenvolver (Controle da Tuberculose, Eliminação da Hanseníase, Controle da $\mathrm{Hi}$ pertensão, Controle da Diabetes M elittus, Ações deSaúdeBucal, Ações deSaúde da Criança eAções de Saúde da Mulher) ${ }^{6}$.

A inclusão da saúde bucal no PSF criou a possibilidade de se instituir um novo paradigma de planejamento e programação da atenção básica e representou a mais importante iniciativa de assistência pública, expandindo e reorganizando as atividades de saúde bucal de acordo com os princípios e diretrizes do SU S?

Considerando que essa expansão é norteada pela organização da lógica assistencial do SUS, os recursos financei ros transferidos na forma de incentivos, através do Piso da Atenção Básica $(P A B)$ variável, destinados à atenção básica por meio das transferências federais a municípios, enfatizam o crescimento de auxílios para a Estratégia Saúde da Família.

De acordo com a Portaria GM /M S no 1.444, de28 de dezembro de $2000^{\circ}$, estabeleceu-seo incentivo financeiro para a reorganização da atenção à saúde bucal prestada nos municípios por meio do PSF; criou-se o incentivo de saúde bucal para o financiamento de ações e da inserção de profissionais de SB; e definiu-se que a equipe de saúde bucal (ESB) trabal haria voltada para a reorganização do modelo de atenção e para a ampliação do acesso às ações de saúde, garantindo-sea atenção integral aos indivíduos eàsfamílias, mediante o estabelecimento de vínculo territorial ${ }^{9}$.

Quanto à incorporação da ESB à Estratégia Saúde da Família, mesmo não estando prevista, nas suas diretrizes, a participação da odontologia, vários municípios acrescentaram-na de diversas maneiras: desde 1995, municípios brasileiros como Curitiba (PR) ${ }^{10}$ e o Distrito Federal ${ }^{7}$ e, no caso de Pernambuco, desde 1998, com Camaragibe ${ }^{11}$, vem agregando à equipe de saúde da fa- 
mília (ESF) a ESB, com iniciativas que vão desde o treinamento dos agentes comunitários de saúde, no sentido de incluírem instruções relativas à saúde bucal em suas atividades, até a inclusão do cirurgião-dentista eseu pessoal auxiliar na equipe da Estratégia de Saúde da Família.

A presenta-se, assim, a evolução da saúde bucal na Estratégia Saúde da Família no estado de Pernambuco como agente ativo das intervenções produzidas pela equi pe de saúde da família, no quetangeàs questões desua implantação para promover, proteger e recuperar a saúde.

\section{Metodologia}

O estudo realizado foi descritivo de caso exploratório e enfoque retrospectivo sobre a saúde bucal no PSF, tendo sido adotada uma abordagem quantitativa, para verificar as características quetal estratégia vem adquirindo com a sua efetiva implantação. 0 universo do estudo constituiu os 185 municípios de Pernambuco, sendo representado por uma amostra, visando à representatividade estadual, com todos os municípios das diferentes regiões do estado, inserido no PSF, que comportaram ESB implantadas.

Todas as informações referentes ao número de municípios com ESF/ESB implantadas, número de ESF/ESB e cobertura populacional foram obtidas a partir dos relatórios repassados mensalmente pelo M S, disponibilizados pela Se cretaria Estadual de Saúde-PE, através da Unidade de Avaliação e Acompanhamento das Ações de Saúde Bucal; como também pelo relatório de competências anuais do D epartamento de Atenção Básica/Secretaria de Aten ção à Saúde/M inistério da Saúde, disponível publicamente no site do MS (www.saude.gov.br).

Os dados sobre os procedimentos odontológicos básicos realizados no estado foram obtidos pelo Sistema de Informação Ambulatorial do Sistema Ú nico de Saúde (Siasus). Tais dados são consolidados e disponibilizados pelo Datasus. 0 evento estudado foi a SB no PSF através da expansão do número deESF/ESB, do aumento no número de procedimentos básicos em saúde bucal. Para demonstrar o quanto o financiamento para atenção básica em saúde bucal promoveu avanço no processo de descentralização, essa variável representou os gastos com saúde bucal (milhões de reais) e sua equivalência proporcional em percentual. 0 período de abrangência do estudo foi de dezembro de 2002 até dezembro de 2005.
Para o método de análise dos dados referentes ao número de municípios com ESF/ESB implantadas, número de ESF/ESB e cobertura populacional, foram construídas tabelas, com base na distribuição das ESF/ESB.

No tocanteà análise dos procedimentos odontológicos da atenção básica, eles foram via dados secundáriosjá agregados e obtidos do Siasus. Tais dados foram explorados e tabulados pelo programa Tabwin 3.2, sendo este um facilitador para o setor de saúde na construção e aplicação de índices e indicadores da produção dos serviços, no planejamento e avaliação de ações para estados emunicípios. A pós o processo de tabulação, os dados coletados foram organizados em planilhas eletrônicas no Excel, sendo os resultados apresentados de forma resumida através de percentagens e proporções.

\section{Resultados}

No final de 2002, o estado de Pernambuco ocupou a 5a posição na região Nordeste (NE) em número de municípios com ESF (172 municípios) ea 8a em número demunicípios com ESB (72 municípios). Quanto ao número deESF edeESB, contabilizaram-se 1.158 e 237, respectivamente, ocupando o 20 lugar em número de ESF e 0 4응 lugar em ESB, mas apresentou a pior relação ESB/ ESF (20,47\%), comparando-se aos outros estados da região (Tabela 1). Esses valores representavam uma cobertura populacional de $20,2 \%$ da ESB, com uma população de 8.084 .722 habitantes (seguindo-se a Portaria GM no 1.444/00) ${ }^{8}$.

Inicialmente, a ESB era referência para duas ESF, então cada ESB cobria, em média, 6.900 pessoas. Essa proporção de 2 ESF:1 ESB representava um fator limitante ao processo de implantação das ESB e impunha aos profissionais um volume de demanda de procedimentos clínicos curativos que comprometia a incorporação da filosofia da Estratégia Saúde da Família no processo de trabalho desses profissionais. Por conseguinte, o percentual de cobertura para ESB era superestimado.

Em 2003, com a Portaria GM no 673/03'12, o M S passou a financiar a ESB na proporção de 1 ESF:1 ESB. Assim, cada ESB passou a cobrir, em média, 3.450 pessoas nos municípios com proporção de 1:1. Desse modo, o cálculo da porcentagem de cobertura da ESB torna-se mais fidedigno. Levando-se em conta a dificuldade de estimar a cobertura da ESB no estado, devido ao fato de a proporção de ESF/ESB não correspon- 
der exatamente ao proposto pelas portarias citadas, e sendo a delimitação atual baseada na Portaria GM no $673 / 03^{12}$ (atendimento de 3.450 habitantes), preferiu-se utilizar, neste estudo, a cobertura de ESB em 29,56\% (população estimada em 8.413.601 habitantes para dezembro de 2005, segundo o Datasus). Por fim, salientamos que, ao se basear na delimitação inicial de 6.900 habitantes com a mesma população, tal percentual de cobertura da ESB fica superestimado (59\%). $\mathrm{N}$ ão se pôde comparar as coberturas ao final de 2002, de 2003 e de 2004 com a cobertura ao final de 2005, pois em 2002 o cálculo era baseado na antiga portaria.
Em dezembro de 2005, Pernambuco ocupou a 6 a posição no $\mathrm{NE}$ em número de municípios com ESF (182). Quanto ao número demunicípios com ESB, mostrou-se uma expansão para 146 municípios (Tabela 1). Também apontou-se Pernambuco ocupando a 2 a posição em crescimento do número de ESF (1.547) e a 6a em número de ESB (721), mas, ainda assim, o estado apresentou a menor relação ESB/ESF (46,61\%).

Ao analisar a expansão e a implantação da saúde bucal na Estratégia Saúde da Família de dezembro de 2002 até dezembro de 2005, verificou-se um favorável crescimento no número de ESB, bem como de ESF (Tabela 2).

Tabela 1. Estados da região Nordeste com percentual decrescente de municípios com Equipes de Saúde da Família (ESF), Equipes de Saúde Bucal (ESB) e relação ESB/ESF em 2002 e 2005.

\begin{tabular}{lrrlrrr}
\hline Municípios c/ ESF & $\mathrm{n}$ & \multicolumn{1}{l}{$\%$} & Municípios c/ ESB & $\mathrm{n}$ & $\%$ & ESB/ESF \\
\hline 2002 & & & & & & \\
PI & 217 & 97,7 & PI & 201 & 90,5 & 39,63 \\
AL & 99 & 97,1 & CE & 158 & 85,9 & 40,93 \\
CE & 175 & 95,1 & PB & 185 & 83 & 23,90 \\
PB & 210 & 94,2 & AL & 84 & 82,4 & 33,61 \\
PE & 172 & 93 & RN & 117 & 70,1 & 20,47 \\
SE & 69 & 92 & SE & 45 & 60 & 29,05 \\
RN & 141 & 84,4 & PE & 72 & 35,1 & 25,14 \\
M A & 135 & 62,2 & M A & 72 & 33,2 & 37,88 \\
BA & 190 & 45,6 & BA & 98 & 23,5 & 33,85 \\
2005 & & & & & & \\
PB & 223 & 100 & PI & 221 & 99,1 & 49,89 \\
CE & 183 & 99,5 & RN & 165 & 98,8 & 85,40 \\
RN & 166 & 99,4 & CE & 182 & 98,7 & 75,00 \\
PI & 221 & 99,1 & PB & 217 & 97,3 & 61,25 \\
AL & 101 & 99 & AL & 98 & 96,1 & 69,70 \\
PE & 182 & 98,4 & M A & 190 & 87,9 & 46,61 \\
M A & 213 & 98,2 & SE & 64 & 85,3 & 84,97 \\
SE & 72 & 96 & PE & 146 & 78,9 & 63,07 \\
BA & 370 & 88,7 & BA & 277 & 66,4 & 54,94 \\
\hline
\end{tabular}

Fonte: Área Técnica de Saúde Bucal/Secretaria deAtenção à Saúde/M inistério da Saúde.

Tabela 2. Evolução das equipes (ESB e ESF), proporção ESB/ESF, distribuição e proporção dos municípios com ESB e ESF. Pernambuco, 2002-2005.

\begin{tabular}{|c|c|c|c|c|c|c|c|c|}
\hline \multirow{2}{*}{ Ano } & \multirow{2}{*}{$\begin{array}{c}\mathrm{ESB} \\
\mathrm{n}\end{array}$} & \multirow{2}{*}{$\begin{array}{c}\text { ESF } \\
n\end{array}$} & \multirow{2}{*}{$\begin{array}{c}\mathrm{ESB} / \mathrm{ESF} \\
\%\end{array}$} & \multicolumn{2}{|c|}{ Municípios c/ESB } & \multicolumn{2}{|c|}{ Municípios c/ESF } & \multirow{2}{*}{$\begin{array}{c}\text { Municípios c/ } \\
\text { ESB/ESF } \\
\%\end{array}$} \\
\hline & & & & $\mathrm{n}$ & $\%$ & $n$ & $\%$ & \\
\hline 2002 & 237 & 1.158 & 20,47 & 72 & 35,1 & 172 & 93,0 & 41,86 \\
\hline 2003 & 310 & 1.263 & 24,54 & 97 & 45,9 & 175 & 94,6 & 55,43 \\
\hline 2004 & 486 & 1.397 & 34,7 & 114 & 56,2 & 178 & 96,2 & 64,05 \\
\hline 2005 & 721 & 1.547 & 46,61 & 146 & 78,9 & 182 & 98,4 & 80,22 \\
\hline
\end{tabular}

Fonte: Área Técnica deSaúde Bucal/Secretaria deAtenção à Saúde/M inistério da Saúde. 
Verificou-se que, apesar da expansão no número deESF eESB, a proporção de ESB/ESF implantadas em Pernambuco ainda não conseguiu alcançar 50\% (Tabela 1). Para os tipos de modalidadequeclassificam as ESB, observaram-se, em 2005, 687 equipes do tipo I e 34 do tipo II. Verificou-se que a expansão da ESB tipo I se deu mais intensamente que a tipo II, ou seja, a tipo I teve um crescimento de $327,14 \%$, enquanto a tipo II teve de $125,92 \%$. No total, o estado apresentou um percentual de $204,22 \%$ para o aumento das ESB (Tabela 3).

Buscaram-se também outras informações quanto à expansão das ESB em Pernambuco que pudessem auxiliar no diagnóstico da situação da ESB no PSF. Remeteu-setal análiseao Siasus, onde se encontram computados todos os dados de que se podem tirar informações a respeito da produção dos procedimentos odontológicos no período estudado. A Tabela 4 apresenta a produção odontológica das ações básicas (procedi- mentos preventivos e curativos) registradas e a evolução proporcional ao número de ESB.

Observou-se que o percentual de crescimento dos procedimentos odontológicos básicos foi de $19,19 \%$, e o percentual de evolução das ESB foi de $204,22 \%$.

Com relação aos recursos para atenção básica em saúde bucal por parte do MS para Pernambuco, apontaram-se as transferências efetuadas por ano de competência, havendo um aumento para a saúde bucal no estado.

No final de 2002, o percentual de recursos despendidos para SB com relação ao total dos recursos destinados para a atenção básica correspondia a aproximadamente $1,5 \%$. Foi registrado, em 2005, um percentual de 4,9\%; houve um aumento de três vezes em relação à proporção da atenção básica em 2002 (Tabela 5). Em relação a valores fixos, o aumento do incentivo foi dequase cinco vezes de 2002 para 2005. Constatou-se que o repasse de incentivos à saúde bucal evoluiu no período analisado em quase $390 \%$.

Tabela 3. Distribuição das ESB implantadas segundo o tipo de modalidades. Pernambuco, 2002-2005.

\begin{tabular}{|c|c|c|c|c|c|c|}
\hline \multirow[t]{2}{*}{ Ano } & \multicolumn{2}{|c|}{$\begin{array}{l}\text { ESB modalidade I } \\
\text { implantadas }\end{array}$} & \multicolumn{2}{|c|}{$\begin{array}{c}\text { ESB modalidade II } \\
\text { implantadas }\end{array}$} & \multicolumn{2}{|c|}{ Total } \\
\hline & $n$ & $\%$ & $n$ & $\%$ & $n$ & $\%$ \\
\hline 2002 & 210 & - & 27 & - & 237 & - \\
\hline 2003 & 284 & 35,24 & 26 & $-3,7$ & 310 & 30,8 \\
\hline 2004 & 452 & 59,16 & 34 & 30,8 & 486 & 56,8 \\
\hline 2005 & 687 & 52 & 34 & 0 & 721 & 48,35 \\
\hline $2002-2005$ & - & 327,14 & - & 125,92 & - & 204,22 \\
\hline
\end{tabular}

Fonte: Área Técnica de Saúde Bucal/Secretaria deAtenção à Saúde/M inistério da Saúde.

Tabela 4. Evolução percentual da quantidade apresentada de procedimentos odontológicos básicos (POB) e equipes de saúde bucal. Pernambuco, 2002-2005.

\begin{tabular}{|c|c|c|c|c|}
\hline Ano & $\begin{array}{l}\text { Quantidade apresentada POB } \\
n\end{array}$ & $\%$ & $\begin{array}{c}\mathrm{ESB} \\
\mathrm{n}\end{array}$ & $\%$ \\
\hline 2002 & 5.754 .963 & - & 237 & - \\
\hline 2003 & 6.774 .363 & 17,71 & 310 & 30,8 \\
\hline 2004 & 6.245 .297 & $-0,78$ & 486 & 56,8 \\
\hline 2005 & 6.859 .781 & 9,83 & 721 & 48,35 \\
\hline $2002-2005$ & & 19,19 & & 204,22 \\
\hline
\end{tabular}

Fonte: M inistério deSaúde/Sistema deInformação Ambulatorial do SUS/Siasus/PE. 
Tabela 5. Transferências para atenção básica, incentivo e percentual do repasse à saúde bucal e evolução em percentual do incentivo à saúde bucal por ano de competência. Pernambuco, 2002-2005.

\begin{tabular}{lcccc}
\hline \multicolumn{1}{c}{ Ano } & $\begin{array}{c}\text { Atenção básica (total) } \\
(\mathrm{R} \$)\end{array}$ & $\begin{array}{c}\text { Incentivo à SB } \\
(\mathrm{R} \$)\end{array}$ & $\%$ & $\begin{array}{c}\text { Evolução do incentivo } \\
(\%)\end{array}$ \\
\hline 2002 & $229.033 .783,64$ & $3.364 .916,46$ & 1,5 & - \\
2003 & $248.234 .348,53$ & $4.549 .849,86$ & 1,8 & 35,21 \\
2004 & $300.322 .729,08$ & $10.060 .650,00$ & 3,3 & 121,12 \\
2005 & $334.597 .316,76$ & $16.471 .300,00$ & 4,9 & 63,72 \\
$2002-2005$ & - & - & - & 389,50 \\
\hline
\end{tabular}

Fonte: M inistério de Saúde/Sistema de Informação Ambulatorial do SU S/Siasus/PE.

\section{Discussão}

Embora tenha sido rotulado de programa, o PSF possui especificidades que fogem à concepção usual dos demais programas do MS, já que não é uma intervenção vertical e paralela às atividades dos serviços de saúde. O PSF possibilitou a integração, promoveu a organização das atividades em um território definido, com o propósito de enfrentar e resolver os problemas identificados; desenvolveu ações de promoção, prote ção erecuperação da saúdee reorientou o modelo assistencial a partir da atenção básica, em conformidade com os princípios do SUS.

Pode-se verificar que, mesmo com a mudança de governo federal e, por conseguinte do ministro de Estado da Saúde, o PSF não sofreu solução decontinuidade, tendo-se, inclusive, expandido em Pernambuco no período estudado.

Os resultados apresentados anteriormente demonstram que, apesar da Portaria GM № 673/ $2003^{12}$, ainda não existe equiparação entre o número de ESB com ESF em Pernambuco. Considerou-se também a ocorrência da defasagem na formação dos recursos humanos de nível médio na área de saú de bucal, especificamente os técnicos em higiene dental (THD), fato averiguado pelo baixo percentual de evolução das equipes tipo II em relação ao tipo I.

Esforços para melhorar os níveis de saúde bucal no estado devem perseguir duas estratégias: a incrementação de medidas preventivas coletivas e a incorporação de pessoal auxiliar odontológico, especialmente o THD, nas ESB. A incorporação de pessoal auxiliar émedida imprescindível para aumentar a cobertura odontológica em níveis coletivo e individual ${ }^{13}$, pois não se pode admitir que o cirurgião-dentista trabalhe no SU S de maneira tradicional (consultório par- ticular), pois isso trará para o SUS uma resposta insuficienteeincapaz principalmente para a maioria da população, que tem, no nível primário da assistência, sua única possibilidade de acesso.

A pós 12 anos do SUS, éfato conclusivo quea expansão efetiva da oferta de serviços para áreas até então desassistidas promoveu mudanças no modelo vigente ${ }^{14}$. Em outro momento, o mesmo autor expõe a dificuldade de persistência do modelo anterior: a superposição da oferta de algumas ações, insuficiência de outras e pouca integração entre os serviços.

Outros autores ${ }^{15}$ contradisseram a afirmação que inicia o parágrafo anterior, argumentando que, mesmo o PSF tendo grande influência nos mecanismos de promoção da saúde e prevenção de doenças, sua capacidade de alteração do modelo assistencial não tem alcançado 0 mesmo sucesso; ou mesmo que o PSF não garantiu, de forma sistemática, o acesso de sua clientela aos níveis de maior complexidade da saúde, nem a universalização da cobertura.

Estudos no Paraná16 demonstraram que os municípios que implantaram atenção à saúde bucal no PSF atéo primeiro semestre de 2002 corresponderam ao total de 278 ESF e $105 \mathrm{ESB}$, sendo 66 modalidade I e 39 modalidade II. Identificou-se também o padrão de maior adesão por parte dos municípios de pequeno porte demográfico, contrapondo o queseverificou no ano de 2000, quando a ESB no PSF passou a ter maior adesão dos municípios de médio egrande portes. Com relação à cobertura, a média da população coberta por equipe correspondia ao mínimo estipulado pela Portaria GM no 1.444/00 do M S8.

Alguns achados explanaram que, para o PSF, em municípios pequenos, altas coberturas populacionais (e consequentemente maior incentivo financeiro por equipe) podem ser obtidas com 
instalação de pequeno número de equipes, significando, para muitos deles, expansão de serviços básicos ${ }^{17}$. Pernambuco apresentou-se da mesma maneira, com expansão das ESB e ESF e da cobertura - consequentemente, da expansão dos serviços básicos. Os referidos autores também afirmaram que existem algumas barreiras a serem rompidas para transpor no modelo de atenção tradicional, em decorrência da formação profissional tecnicista e hospital ocêntrica, pois o cirurgião-dentista e sua equipe estão basicamente envolvidos em atividades clínicas, não tendo tempo de atuar com a família, como o próprio nome diz, deixando de ser PSF.

As mudanças culturais constituem os maiores obstáculos a serem vencidos, principalmente no que se refere à odontologia. No entanto, é necessário que tais mudanças se façam em ritmo mais acelerado, pois a saúde bucal não pode se atrasar mais ainda, no processo de transformação do modelo de atenção em saúde prestado à população brasileira.

Ao contrário do que já foi afirmado ${ }^{7,13,18}$ sobre o período anterior à inclusão da saúde bucal no PSF, partimos do pressuposto embasado nos dados para Pernambuco, de dezembro de 2002 até dezembro de 2005, de que ocorreu uma mudança na práxis da atenção básica em odontologia: antes eram evidenciados os procedimentos curativos de ampla demanda espontânea; passou-se então a se ofertar um maior número de procedimentos preventivos.

Estudo anterior ao modelo de atenção em saúde bucal nos municípios pernambucanos ${ }^{19}$ concluiu que $57 \%$ dos municípios produziram uma atenção básica composta em mais de 50\% por procedimentos curativos, caracterizando 0 modelo de atenção em saúde bucal em Pernambuco como predominantemente curativo - fato contraditório aos resultados apresentados para o período analisado.

Pesquisa realizada com dentistas ${ }^{16}$ revelou que, ao serem questionados sobre os tipos de procedimentos executados pela equipe, eles alegaram que a falta de ações preventivas e de promoção de saúde se dá pela precariedade socioeconômica da população que necessita de numerosos tratamentos, e os profissionais sentem a necessidade de adequar a oferta dos serviços à realidade local para melhoria do acesso.

Confrontando tal assertiva, talvez em alguns municípios do estado isso não venha a contribuir para uma mudança operacional no acesso à assistência odontológica e na atenção à saúde bucal, pois a incorporação das ações de saúde bucal no PSF confirma o princípio da integralidade das ações, passando de um modelo centrado no alívio da dor para um modelo baseado em ações de promoção e recuperação da saúde bucal, bem como de prevenção de doenças.

Analisando-se a Tabela 3, nota-se que houve uma evolução na quantidade apresentada de procedimentosodontológicos básicos(POB) de 2002 a 2005. Salienta-se que o quantitativo de POB apresentado pelo Siasus ainda não é o real, pois existe subnotificação no sistema, quegera inconsistências nos registros do banco. Grosso modo, observa-se, no total, que o percentual de crescimento dos procedimentos ano a ano foi positivo (19,19\%), porém esse percentual não acompanhou proporcionalmente o percentual de evolução das ESB no estado (204,2\%).

$N$ ão se conseguiu averiguar nem identificar os motivos pelos quais, no ano de 2003, houve um percentual negativo no quantitativo de $P O B$, registrado no Siasus. Observa-se que ainda há resistência em notificar as informações, o que compromete o registro dos dados, ocasionando a subnotificação, como já dito anteriormente.

Uma primeira avaliação sobre o PSF apontou para o impacto positivo do programa em áreas com grande déficit de serviços básicos de saúde, sobretudo nas regiões N orte e N ordeste do país ${ }^{20}$. Todavia, um dos grandes desafios do programa se refere ao seu impacto nas grandes regiões metropolitanas, onde, ao menos em tese, há uma oferta mais estruturada de serviços de saúdee ondese concentra um expressivo contingentepopulacional com elevado índicede pobreza urbana e, ao mesmo tempo, extremamente heterogênea.

Vale ressaltar também que a ESB do PSF deve trabal har na perspectiva da vigilância em saúde bucal, a qual não faz imposição de troca do modelo de atenção à saúde vigente do ponto de vista assistencial, porém no que diz respeito aos aspectos do quadro sanitário da população. Tais aspectos deverão ser modificados.

Em conformidade com a opinião pelo qual o cirurgião-dentista da equipe não pode continuar trabalhando de forma isolada, tanto na sua atuação quanto no seu saber, ele deve fazer parte da estruturação dos serviços de saúde, promovendo uma relação mais próxima para prestar assistência integral eresolutiva a toda a população, intervindo de forma apropriada ${ }^{21}$.

Em um estado onde os municípios são caracterizados por apresentarem alto nível de desigual dade de renda e de capacidade de gerar receitas próprias, os recursos federais cumprem um 
papel fundamental na busca da equidade dos gastos do PSF. Confirma-se, assim, a assertiva de que poucos estados brasileiros têm destinado recursos aos seus municípios para a instalação da Estratégia Saúde da Famíliaํ․

A partir da efetivação e do incentivo financeiro à ESB ao final de 2000, 0 acesso da população às ações de saúde bucal ampliou-se, as práticas foram orientadas consoante o preconizado pelo PSF, bem como o envolvimento de gestores (estaduais e municipais) contribuiu para a real implantação do programa.

A promulgação do SUS desencadeou, no estado, um processo rápido e marcante de descentralização, com maior autonomia técnica efinanceira decorrente do repasse da totalidade dos re cursos para alguns municípios (habilitados se gundo o tipo de gestão).

Há uma percepção de que o financiamento da saú de bucal contribuiu para a diluição da cultura do clientelismo, encerrando contradições importantes como "assistência à saúde para pobres" ou "catalisador de mudanças na qualidade dos serviços", ou ainda um apelo para obtenção de recursos financeiros, confirmando estudos anteriores ${ }^{22}$.

Entretanto, $\mathrm{M}$ arques e $\mathrm{M}$ endes $\mathrm{s}^{15} \mathrm{e}$ Baldani et al. ${ }^{16}$ corroboraram a ideia de que a implantação do PSF aumentou os recursos do MS alocados à atenção básica, transferidos mediante o PAB variável, o qual passou a incorporar diferentes incentivos que premiam os municípios que desenvolvem os programas sugeridos. Concorda-se com esses autores quanto ao financiamento do programa (estratégia), quando o apontam como problemático einstável, principalmenteno ponto devista dos municípios, uma vez queo volumede recursos de origem municipal passível a ser destinado ao programa é muito variável e geralmente escasso - o que não ocorre com os incentivos federais (Portaria GM no 673/03) 12 $^{12}$ destinados às ações de saúde bucal no âmbito do PSF, parte integrante do PAB (valor anual pago por equipe modalidade I: $R \$ 20.400,00$; e modalidade II: $R \$$ $26.400,00$ ), que são fixos e programados.

Ainda dando ênfase ao financiamento, estudos com coordenadores estaduais e secretários municipais de saúde de Santa Catarina revelaram que uma das principais limitações para operacionalização do PSF seria decorrente da falta de recursos financeiros e de entendimento por parte dos gestores ${ }^{22}$. Enfocando a NOB $96^{23}$, as transferências federais per capita para os municípios foram, a cada ano, mais interessantes e contribuíram para mudanças no perfil da atenção básica.
Sobre o relatado, indicou-se que um grande desafio do programa (estratégia) diz respeito à sua capacidade de integração com o restante do sistema de saúde, de forma a redefinir qualitativamenteo modelo de atenção à saúde, como pretendido por seus formuladores.

Salienta-se a preocupação de quea Estratégia Saúde da Família possa constituir-se em um mecanismo de focalização dentro do princípio da universalização dos direitos promovido pelo SUS, trazendo outro grande desafio de compatibilizar o estabelecimento de prioridades sem adotar mecanismos de restrição de direitos sociais, mostrando que não é apenas uma proposta de atenção "básica", simplificada e barata para áreas e população pobres ou uma cesta básica para o setor, como apregoou o Banco M undial.

\section{Consideraçõesfinais}

N este estudo, verificaram-se alguns aspectos sobre a implantação da saúde bucal na Estratégia Saúde da Família em Pernambuco. Pelo exposto, Pernambuco ocupava uma posição privilegiada em relação aos outros estados do NE tanto no número de municípios com ESF eESB implantadas quanto no número de ESF e ESB - apesar de apresentar uma relação ESB/ESF menor que $50 \%$, ou seja, o estado não conseguiu a equiparação de

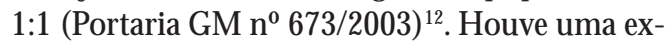
pansão no número de equipes de saúde bucal de $204,22 \%$ entre dezembro de 2002 e dezembro de 2005. Pôde-seconcluir queo crescimento da modalidadel em relação à II ocorreu ativamentepela carência deprofissional denível médio (THD) na ESB, fazendo-se necessária a formação de THD em todos os municípios, principalmente no interior do estado. Pôde-se afirmar que o percentual de evolução dos procedimentos odontológicos básicos realizados no período estudado éincompatível com o percentual de expansão do número de ESB, o que leva a se acreditar que, na prática, os serviços existentes de assistência odontológica para atenção básica foram transformados em equipes de saúde bucal.

Os esforços do governo federal para consolidar a Política Nacional de Saúde Bucal (Brasil Sorridente) maximizaram os incentivos repassados à saúde bucal em torno de $390 \%$. Foi possível compreender que a Estratégia Saúde da Família, de fato, evolui de um programa isolado para uma estratégia de reorientação do modelo assistencial na rede pública, havendo concordância quanto à visi bilidade de sua trajetória e efei- 
tos positivos na integralidade. Por meio dos resultados deste estudo, pode-se observar que os principais problemas e dificuldades vivenciados pela ESB não são exclusivos dos municípios pernambucanos. Pretende-se com este estudo estimular o desenvolvimento de novos trabal hos com esse tema, para que possam contribuir para a consolidação da saúde bucal na Estratégia Saúde da Família.

\section{Colaboradores}

SF Silva organizou o banco de dados, fez a concepção e o delineamento do estudo, análise e interpretação dos resultados e a redação do artigo; PJL M artelli orientou o delineamento do estudo, participou da análise e da discussão dos resultados e da revisão final; DA Sá contribuiu com a tabulação e a análise dos dados do Siasus; AP Cabral, FC Pimentel eISM onteiro eCLSV M acedo contribuíram na discussão dos achados e na revisão final.

\section{Agradecimentos}

Estetrabalho contou com o apoio daÁrea Técnica de Saúde Bucal do M inistério da Saúde e da Unidade de Acompanhamento e Avaliação das Ações de Saúde Bucal da Secretaria de Saúde do Estado de Pernambuco. 
1. Marques RM, M endes A. O SU S e a atenção básica: a busca de um novo desenho? [site da Internet] [acessado 2005 out 26] 2001 [cerca de 17 p.]. Disponível em: http://www.opas.org.br/servico/arquivos/Sala5415. pdf

2. Cordón JA. Sobre a construção histórica do conceito de odontologia em saúde coletiva. Ação Col 1999; 1:7-25.

3. Brasil. Lei $n^{\circ} 8.080$, de 19 de setembro de 1990. Dispõe sobre a promoção, proteção e recuperação da saúde, a organização e o financiamento dos serviços correspondentes e dá outras providências. Diário Oficial da União 1990; 20 set.

4. Brasil. M inistério da Saúde. Secretaria de Gestão Participativa. Saúde da Família: panorama, avaliação e desafios. Brasília: M inistério da Saúde; 2005.

5. Souza DS, Cury JA, Caminha JAN, Ferreira MA, Tomita NE, Narvai PC, Gevaerd SP, Mendes WB. A inserção da saúde bucal no PSF. Rev Bras Odonto Saúde Col 2001; 2:13-29.

6. Brasil. M inistério da Saúde. Portaria GM n 95. Regionalização da assistência à saúde: aprofundando a descentralização com equidade no acesso: N ormas O peracionais de Assistência à Saúde. Brasília: Diário Oficial da União 2001; 26 jan.

7. Zanetti CHG. Por um caminho sustentável para universalização da atenção básica: saúde bucal da família com equidade e integralidade. Brasília: UnB; 2000. [acesso 2005 out 4]. [cerca de 9 p.] Disponível em: http:// www.saudebucalcoletiva.unb.br/oficina/estado/ coletivo_amplo/psf_caminho.htm

8. Brasil. Portaria $n^{0}{ }^{-}$1.444. Estabelece incentivo financeiro para a reorganização da atenção à saúde bucal prestada nos municípios por meio do Programa de Saúde da Família. Diário Oficial da União 2000; 28 dez.

9. Brasil. Ministério da Saúde. Portaria GM n 1.444 . Estabelece incentivo financeiro para a reorganização da atenção à saúde bucal prestada nos municípios por meio do Programa de Saúde da Família. Brasília: Diário Oficial da União 2000; 28 dez.

10. Roncalli AG, Arcieri RM, Unfer B, Costa ICC, M oraes E, Saliba NA. M odelos assistenciais em saúde bucal no Brasil: tendências e perspectivas. Ação Col 1999; 1:8-14.

11. Martelli PJL. Análise da atenção em saúde bucal no nível municipal: caracterização do modelo assistencial [dissertação]. Recife: Centro de Pesquisas Aggeu M agalhães, Fundação Oswaldo Cruz; 2001.

12. Brasil. M inistério da Saúde. Portaria no 673/GM . Publicada no Diário Oficial da União. Atualiza e revê 0 incentivo financeiro às Ações de Saúde Bucal, no âmbito do Programa de Saúde da Família, parte integrante do Piso de Atenção Básica-PAB. Diário Oficial da União 2003; 4 jun.

13. Frazão P. Tecnologias em saúde bucal coletiva. In: Botazzo C, Freitas SFT, organizadores. Ciências sociais e saúde bucal: questões e perspectivas. São Paulo: Unesp; 1998. p. 159-175.
14. Roncalli AG. O desenvolvimento das políticas públicas de saúde no Brasil e a construção do SUS. In: Pereira AC, organizador. O dontologia em Saúde Coletiva: planejando ações e promovendo saúde. Porto Alegre: Artmed; 2003. p. 28-49.

15. Marques RM, M endes $A$. A política de incentivos do M inistério da Saúde para atenção básica: uma ameaça à autonomia dos gestores municipais e ao princípio da integralidade? Cad Saude Publica 2002; 18(Supl.):163-171.

16. Baldani $M H$, Fadel $C B$, Possamai $T$, Queiroz M GS. A inclusão da odontologia no Programa de Saúde da Família no estado do Paraná, Brasil. Cad Saude Publica 2005; 21:1026-1035.

17. M edina MG, Aquino R. Avaliando o Programa Saúde da Família. In: Sousa MF, organizador. Os sinais vermelhos do PSF. São Paulo: Hucitec; 2002. p. 135151.

18. Trad LAB, Bastos ACS. 0 impacto sociocultural do PSF: uma proposta de avaliação. Cad Saude Publica 1998; 2:429-435.

19. Oliveira LMA. Saúde bucal em Pernambuco: acesso e utilização dos serviços odontológicos do SUS em 2001 [monografia]. Recife: Centro de Pesquisas Aggeu Magalhães, Fundação Oswaldo Cruz; 2002.

20. Senna MCM. Equidade e política de saúde: algumas reflexões sobre o Programa Saúde da Família. Cad Saude Publica 2002; 18 (Supl.):203-211.

21. Rosa WAG, Labate RC. Programa Saúde da Família: a construção de um novo modelo de assistência. Rev Latino-Am Enfermagem [periódico na Internet]. 2005 nov-dez [acessado 2006 mar 14]: 6:1027-1034. [cerca de 8 p.]. Disponível em: http://www.scielo.br/pdf/ rlae/v13n6/v13n6a16.pdf.

22. Conill EM. Políticas de atenção primária e reformas sanitárias: discutindo a avaliação do Programa de Saúde da Família em Florianópolis, Santa Catarina, Brasil, 1994-2000. Cad Saude Publica 2002; 18(Supl.):191-202.

23. Brasil. M inistério da Saúde. NOB-SUS 1996. Norma Operacional Básica do Sistema Ú nico de Saúde-SUS. Gestão plena com responsabilidade pela saúde do cidadão, Brasília, janeiro/1997. Braślia: Ministério da Saúde; 1997. (Publicada no D.O.U. de 6/11/1996) [Documento da Internet]. 2006 [acessado 2006 mar 14]. Disponível em: http://siops.datasus.gov.br/ Documentacao/N OB\%2096.pdf

Artigo apresentado em 27/12/2007

Aprovado em 14/03/2008

Versão final apresentada em 14/04/2008 